\title{
Hamlet et la Préface de Marie de Gournay
}

RICHARD

HILLMAN

Résumé: Vers 1600, Shakespeare devait se mettre à remanier une pièce démodée sur le sujet de Hamlet, en puisant, d'après un grand nombre de spécialistes, dans les Essais de Montaigne. Pourtant la Préface de Marie de Gournay à l'édition de 1595 des Essais n'a jamais été mise en rapport avec l'oeuvre shakespearienne. Bien qu'il n'y ait eu aucun lien démontrable entre ces deux textes, une approche franchement intertextuelle nous permet de considérer celui de de Gournay comme une sorte d'activeur de certains éléments montaigniens, surtout en ce qui concerne la transformation de la situation familiale de Hamlet en drame d'identité psychologiquement surchargé, qui met en cause les catégories du masculin et du féminin.

$\mathrm{P}$

our ceux et celles qui croient, comme moi, ${ }^{1}$ que la théorie intertextuelle peut fournir un moyen innovateur et producteur de rapprocher des textes n'ayant aucun rapport démontrable au niveau de l'"influence," le cas de Hamlet (environ 1600) et de la Préface de Marie de Gournay à l'édition de 1595 des Essais de Montaigne pourrait sembler avoir été fait exprès pour servir d'exemple idéal. D'une part, le lecteur, de 1600 ou de notre époque, est susceptible, comme je tiens à le montrer plus bas, d'être frappé par de nombreux parallèles entre ces deux textes en ce qui concerne des mots, des idées, et surtout certains éléments psychologiques. D'autre part, ces parallèles ne suffisent nettement pas à eux seuls pour qu'un lien direct soit établi, d'autant plus que les enjeux sont énormes, du point de vue de l'histoire littéraire et même culturelle. Car un argument en faveur de l'influence de la Préface dans ce cas entraînerait forcément la suggestion que le chef-d'oeuvre capital du premier écrivain anglais, sinon mondial, dont la génie incomparable reste une 
donnée jusqu'à présent, ait été fondamentalement inspiré par l'ouvrage mineur, soi-disant supplémentaire, d'une Française assez obscure. Tout cela sent l'absurdité. Sans l'intertextualité, il faudrait donc se taire en face des deux textes réciproquement provocateurs. Dans les pages suivantes, je propose une alternative à un tel silence, laquelle se contentera de montrer à quel point ces discours divergents font néanmoins partie du même "espace discursif," ce qui toutefois servira à élaborer la définition d'un moment historique et culturel franchissant des frontières d'une façon peut-être inattendue.

Commençons par mettre en relief quelques mots tirés de Hamlet, lesquels, au premier regard, ont l'air tout à fait normaux, sinon banals, étant donné la misogynie répandue à l'époque. Il s'agit de la dernière scène du drame, au moment où le héros partage avec son confident Horatio sa méfiance - bien fondée, évidemment - au sujet de l'invitation du Roi et de Laertes au match d'escrime dissimulant leur complot: "It is but foolery, but it is such a kind of gain-giving, as would perhaps trouble a woman" $(5.2 .215-16){ }^{2}$ Le terme "gain-giving" (ainsi dans le texte du Folio de 1623; les autres éditions contemporaines donnent la variante "gam[e-]giuing") est l'équivalent archaïque de "misgiving" en anglais moderne, seulement plus fort, grâce à la connotation présente dans "gain" (c'est-à-dire "contre") d'un esprit divisé.

Or Hamlet se manifeste misogyne d'un bout à l'autre de la pièce, à peu près dès ses premières paroles. Néanmoins, les reproches violents dont il accable Gertrude, sa mère, ainsi que Ophelia, à laquelle il avait autrefois fait la cour, portent exclusivement sur la prétendue faiblesse des femmes en face de leurs passions et leur vanité: "Frailty, thy name is woman!" (1.2.146). Il n'y a pas de "gain-giving" considéré comme propriété féminine, encore moins lorsqu'il s'accuse dans un de ses soliloques de ressembler à quelque femme vulgaire, proférant des injures au lieu d'agir. Dans l'aveu qu'il fait à Horatio, bien sûr, Hamlet persiste dans sa préoccupation pour la faiblesse féminine. Mais on y remarque aussi une reconnaissance de la femme, bien que cette reconnaissance soit voilée et mêlée de mépris, en tant qu'être complexe, doué de voix qui se contredisent - bref, comme sujet dans le sens psychologique du terme. D'ailleurs, cette image est carrément intériorisée. La femme surgit donc dans la pensée de Hamlet pour la dernière fois non pas comme simple objet d'antipathie, toujours autre, mais plutôt, jusqu'à un certain point, comme son semblable, comme l'origine d'ailleurs de cette voix à l'intérieur de luimême qui a pour but de lui sauver la vie.

Dans ce contexte, la comparaison dont Hamlet se sert pour exprimer son inquiétude perd considérablement sa qualité conventionnelle, sinon sa valeur 
négative. Par conséquent, elle acquiert un caractère exceptionnel dans le discours de Hamlet pour jouer, dans la terminologie de Riffaterre, le rôle d'une "agrammaticalité" signalant la présence d'un intertexte. Ce rôle s'impose d'autant plus que la porte est ainsi ouverte à une intégration de cet aspect féminin du texte aux plus profondes questions d'identité, de subjectivité, de loyauté et de conscience - questions qui sont à leur tour étroitement liées au drame familial de Hamlet, c'est-à-dire à ses relations problématiques avec sa mère et avec le souvenir (qui se mêle au survenir) de son père.

L'engagement de la pièce shakespearienne dans de tels thèmes justifie sa renommée comme monument innovateur du drame philosophique et psychologique. Car la tradition dont Hamlet émerge en 1600, d'une façon tout à fait imprévisible, ne possède pas ces éléments, sinon à l'état embryonnaire. Il s'agit de la tragédie de vengeance, populaire depuis les années quatre-vingts du siècle, mais dix ans plus tard déjà démodée, du moins chez plusieurs intellectuels qui s'en moquaient. Ce genre dramatique est une variante de la catégorie plus large de "tragédie de sang," ce qui souligne son caractère sensationnel et primitif. Shakespeare, semble-t-il, a décidé, vers la fin du siècle, d'en remanier un spécimen assez connu, probablement de Thomas Kyd, auquel on accorde généralement le titre de Ur-Hamlet. Bien que, malheureusement, cette pièce originale n'existe plus, certaines remarques contemporaines, ainsi que les versions non-dramatiques du récit, mènent à croire qu'elle ne s'est pas élevée au-dessus du mélodrame brutal typique de l'époque. Ce n'est pas diminuer les réalisations de Shakespeare que de prendre les divergences radicales qui séparent la nouvelle pièce de ses origines pour un autre signe "agrammatical" de présence intertextuelle.

Il.va sans dire que depuis des centaines d'années de nombreux spécialistes (et non-spécialistes) ont suivi de nombreux fils à la recherche des "sources" des concepts introduits par Shakespeare dans ses matériaux hérités. L'un de ces fils pris en main le plus souvent est celui qui remonte à Montaigne. Comme c'est toujours le cas dans la critique de Shakespeare, une grande partie du champ de l'enquête est occupée par des assertions exagérées et peu convaincantes, qui parfois même se contredisent. Cependant la plupart des shakespeariens de nos jours s'entendent sur la probabilité que l'auteur anglais ait puisé dans les Essais. ${ }^{3}$ Et même s'il ne l'a pas fait directement - ce qui reste pourtant possible, étant donnée sa connaissance démontrée de la langue française - il devait se servir au moins, dit-on, de la traduction de John Florio (éditée en 1603 mais que Shakespeare aurait pu avoir consultée en manuscrit quelques années plus tôt). On met souvent en parallèle, par exemple, le discours dans lequel 
Hamlet se rend compte de son aliénation de la beauté de la création (2.2.297 ff.) et, d'autre part, l'ironie au dépens de l'homme, malgré ses avantages superficiels, que Montaigne développe dans l"'Apologie de Raimond Sebond."

Puisque je m'intéresse non pas aux sources mais plutôt aux intertextes, même les exagérations et revendications floues de certains critiques sont révélatrices. Car celles-ci servent à établir la présence intertextuelle d'un texte, enfin de chacun des deux textes, à l'intérieur de l'autre dans l'esprit de générations de lecteurs. Tout en avouant que j'ai une arrière-pensée, j'aimerais mettre en jeu encore un parallèle qui n'a pas été remarqué, à ce que je sache. Or l'affinité semble être notamment forte entre les attitudes sceptiques de Hamlet et de Montaigne envers l'existence humaine dans un univers où Dieu se cache plus que ce n'était le cas pour les penseurs du Moyen Age. À juste titre, l'"Apologie de Raimond Sebond" est le texte montaignien le plus souvent cité à cet égard. Mais je tiens à en souligner un autre qui paraît d'ailleurs offrir une réponse à la fameuse question posée par Hamlet:

To be, or not to be, that is the question:

Whether 'tis nobler in the mind to suffer

The slings and arrows of outrageous fortune,

Or to take arms against a sea of troubles, And by opposing, end them.

Il est clair par la suite laquelle des deux possibilités est, pour Hamlet, la solution préférable; selon lui, c'est à cause de la réflexion trop curieuse sur les mystères de l'au-delà que "enterprises of great pitch and moment/...their currents turn awry,/And lose the name of action" (85-87). Hamlet et Montaigne s'accordent donc, semble-t-il, sur le principe, énoncé par Montaigne dans le Chapitre 8 du deuxième tome des Essais, que "estre consiste en mouvement et action" (Montaigne, 2: 59), ${ }^{5}$ tandis que la passivité équivaut à une nonexistence indigne. D'ailleurs, selon autant Hamlet que Montaigne, une passivité pareille serait bestiale et déraisonnable, comme en temoignent les passages suivants, mis en parallèle par John M. Robertson, il y a une centaine d'années: ${ }^{6}$

Puisqu'il a pleu à Dieu nous doüer de quelque capacité de discours, affin que, comme les bestes, nous ne fussions pas servilement assujectis aux lois communes, ans que nous nous appliquassions par jugement et liberté volontaire, nous devons bien prester un peu à la simple authorité de nature, mais non pas nous laisser tyranniquement emporter à elle; la seule raison doit avoir la conduite de nos inclinations. 


\begin{abstract}
What is a man,
If his chief good and market of his time

Be but to sleep and feed? a beast, no more.

Sure He that made us with such large discourse,

Looking before and after, gave us not

That capability and godlike reason

To fust in us unused.
\end{abstract}

Notons aussi que ces propos de Hamlet, qui se trouvent dans son soliloque du quatrième acte où il se penche sur l'exemple courageux de Fortinbras, font écho à sa condamnation plus tôt de sa "faible" mère pour avoir rompu les liens d'affection avec son père: "O God, a beast that wants discourse of reason/ Would have mourned longer"'(1.2.150-51). Outre les ressemblances spécifiques entre ces lieux shakespeariens and montaigniens, dans la forme ainsi que dans le contenu, il est frappant que l'essai que je viens de citer soit celui "De L'affection des pères aux enfans."

Mais rapprocher plus largement cet essai de la pièce de Shakespeare c'est préciser la distance qui existe entre eux. D'une part, après avoir affirmé que l'amour des pères envers leurs enfants est plus fort de nature que celui que les enfants éprouvent à leur tour, Montaigne conseille des sentiments paternels plus restreints, basés sur le mérite évident des enfants. D'autre part, Hamlet se reproche précisément, pendant son soliloque du quatrième acte, un manque de passion et de loyauté envers son propre père, qui avait d'ailleurs établi l'acte de vengeance comme véritable pierre de touche de l'amour filial: "If thou didst ever thy dear father love - " (1.5.23). Jusqu'à un certain point, la remarquable concordance verbale des deux textes met en relief leurs perspectives radicalement divergentes: celle du père qui doit juger avec sang-froid, celle du fils qui se sent toujours sous le regard du père.

La relation intertextuelle peut donc nous alerter quant à une divergence analogue au sein du texte shakespearien. Mais il existe aussi une divergence entre les deux textes en ce qui concerne l'élément psychologique, qui est tout à fait manquant chez Montaigne. Pour nous servir en passant de termes lacaniens, on pourrait dire que la pièce transforme la présence surveillante du père en véritable Nom-du-père et montre le fils non seulement comme assujetti au jugement paternel mais aussi comme menacé au niveau de son être, donc redéfini comme sujet dans l'acception psychanalytique du terme, par son incapacité à jouer le fils idéal. Montaigne parle des émotions dans cette relation seulement afin de les déprécier en faveur du devoir. Par contre, Shakespeare 
les valorise comme site de lutte intérieure d'une façon sans précédent. Au bout du compte, l'Essai est loin d'anticiper les mécanismes par lesquels la pièce transforme la situation familiale de Hamlet en drame d'identité psychologiquement surchargé. Il en est ainsi, d'ailleurs, pour les Essais en général, y compris l'"Apologie de Raimond Sebond," malgré le cadre thématique qu'ils semblent souvent fournir, même malgré les éléments dans l'Apologie qui portent sur les relations de l'auteur avec ses pères terrestre et divin. ${ }^{8} \mathrm{Au}$ sein de cette dynamique intertextuelle, il y a donc quelque chose d'inachevé, quelque "agrammaticalité" qui signale la présence d'un autre intertexte. De plus, nous n'avons pas encore de femme dans le tableau.

Voilà que la Préface de Marie de Gournay s'impose comme supplément, s'interpose entre Shakespeare et Montaigne. Cathleen M. Bauschatz a récemment développé l'idée que la Préface sert de sorte de supplément féminin aux Essais, en tant que les derniers - Bauschatz met elle aussi l'accent sur l'Apologie - traitent dans une perspective spécifiquement masculine d'un "struggle for self-definition in relation to the father, who is revered and yet who causes anxiety." Avant Bauschatz, le commentaire indispensable de François Rigolot, qui a préparé la seule édition moderne de la Préface, avait déjà établi à quel point l'aspect psychologique et la perspective féminine sont évidents, voire dominants, dans l'ouvrage de de Gournay. Il s'agit franchement, pour la soi-disant "fille d'alliance" de Montaigne, d'un vrai drame familial. Selon Rigolot, la lutte signalée par Bauschatz engendre le "double discours" de la Préface: d'une part, "la voix féminine ne saurait s'exprimer sans avoir constamment à se justifier aux yeux de ses lecteurs virtuels"; d'autre part, puisque "le 'père' demande à sa 'fille' de parler pour lui et non pour elle, c'est seulement à travers les intérêts du surmoi paternel que peut se faire reconnaître l'identité de la 'fille d'alliance'."10 Voilà un exemple net de "such a kind of gain-giving, as would perhaps trouble a woman," basé sur un modèle psychologique fort semblable à celui qui a été souvent invoqué pour expliquer la force motrice (ou plutôt immobilisante) du personnage shakespearien, qui se sent incapable ou de remplir ou de laisser tomber le rôle imposé par son père.

Même la double dimension de ce rôle, qui est vindicatif du point de vue personnel et en même temps revendicatif, en ce qui concerne le trône danois et la justice, a son équivalent dans la défense entreprise par de Gournay au commencement de la Préface à la fois contre les détracteurs des Essais et, plus personnellement, contre ceux, parmi ses propres lecteurs, qui méprisent les femmes sous prétexte de leurs multiples faiblesses. Dans l'imagination de Marie de Gournay aussi ne s'agit-il pas d'une mission confiée par un père 
revenant de la mort, ce qui est mis en relief par les propos de Rigolot: "Cette 'liaison,' qui se poursuit au-delà de la mort du père pour déterminer l'identité de la fille, constitue ainsi à la fois une promesse de plénitude et un danger d'aliénation"? ' Rien dans la représentation des relations filiales chez Montaigne ne correspond à la très vive clarté avec laquelle de Gournay rend hommage à la veuve de Montaigne parce que cette dernière a "voulu r'embrasser et r'échauffer en moy les cendres de son mary, et non pas l'espouser mais se rendre une autre luy-mesme"(26). ${ }^{12}$ Rien non plus dans les Essais, dont le but déclaré est de faire l'autoportrait, ne nous prépare à un tel effacement par de Gournay de sa propre image - "je ne suis moy-mesme que par où je suis sa fille" (25) - bien que cet effacement se déguise en affirmation assurée, sinon présomptueuse:

...la nature m'ayant faict tant d'honneur que, sauf le plus et le moings, j'etois toute semblable à mon Pere, je ne puis faire un pas, soit escrivant ou parlant, que je ne me trouve sur ses traces; et croy qu'on cuide souvent que je l'usurpe (46).

$\mathrm{Au}$ niveau de l'influence littéraire, certaines expressions extrêmes employées par de Gournay pour décrire la dépendance de son identité de celle de son "père" s'expliquent assez facilement par le modèle des Essais. Comme le constate Rigolot, ${ }^{13}$ l'auteur puise librement dans le récit de Montaigne de sa parfaite amitié avec La Boétie et de son propre deuil après la mort de ce dernier. Il semble que de Gournay cherche à se modeler dans une certaine mesure sur les deux personnages dans cette situation: elle aurait pu finir par occuper dans les affections de Montaigne une place pareille à celle de La Boétie; à présent, par contre, elle souffre comme Montaigne l'avait fait. Elle souffre même plus, car elle dépasse sa propre déclaration que "Estre seul c'est n'estre que demy," laquelle est modelée sur Montaigne ("il me semble n'estre qu'à demy" [1: 210]), pour affirmer que le survivant d'une telle amitié " $n$ 'est plus que par son mal-heur" (50).

Être amie pour Marie de Gournay est inséparable de l'idée d'être fille:

Estre amy c'est n'estre que depositaire de soy-mesme. La plus grande infelicité du monde c'est d'avoir la plus grande felicité; je l'avois en ce tres grand-Pere, puis qu'il en fault achepter la possession terminée au prix de la privation perpetuelle (51, nous soulignons).

Paradoxalement, l'effet de l'intertexte montaignien est de déstabiliser une conception extrêmement mélangée du soi. Car Montaigne, pour sa part, commence son éloge de La Boétie en distinguant assez brutalement entre l'amitié et l'attachement sentimental d'un enfant pour son parent, en limitant 
cette dernière relation au respect et en la soupçonnant de dégénérer en méfiance et en violence à cause des intérêts, des identités mêmes, fatalement concurrentiels:

Il s'est trouvé des nations où, par usage, les enfans tuoyent leurs peres, et d'autres où les peres tuoyent leurs enfans, pour eviter l'empeschement qu'ils se peuvent quielquefois entreporter, et naturellement l'un depend de la ruine de l'autre (1: 199).

Lorsqu'il s'agit de l'enfant et du parent, se regarder comme "perdu en soy, pour se recouvrer en autruy" (de Gournay 51), comme le fait de Gournay, est donc forcément mettre en relief l'ambivalence inhérente à l'expérience d'une telle perte. Or c'est précisément une pareille ambivalence qui caractérise le deuil obsessionnel de Hamlet, qui également, du moins avant l'imposition du devoir concret par l'apparition de son père, "n'est plus que par son mal-heur."

À part le fait que le deuil de Montaigne n'occupe qu'une part très limitée de son ouvrage à mille facettes, le remaniement de cet élément par Marie de Gournay souligne une distinction plus générale entre elle et Montaigne, laquelle rapproche encore la Préface de l'oeuvre shakespearienne. Que ce soit par rapport à La Boétie ou à Montaigne, c'est ainsi par comparaison à d'autres que de Gournay s'engage dans la construction de son identité. L'éloge de Montaigne ne contient pas cet élément, du moins non pas dans la même forme. Bien sûr, on a beaucoup parlé récemment de la construction du sujet chez Montaigne, en appliquant parfois l'idée du texte comme miroir, ou plutôt comme série de miroirs. ${ }^{14}$ Dans ce système, la comparaison de l'auteur avec d'autres personnages, qu'ils soient tirés de la connaissance personnelle, de l'histoire, ou de la mythologie, joue un rôle important. Mais je mettrai l'accent sur le mot “joue,"en proposant qu'il s'agit d'un texte qui s'empare du privilège d'improviser, qui franchement suspend une identité bien établie aux yeux des autres afin de la recréer par fragments. Ce qui présume, sinon la stabilité de l'écriture même, pour le moins une confiance fondamentale en le droit de l'écrivain d'écrire. C'est précisément cette confiance qui manque chez Marie de Gournay, pour qui la tiraillante question, équivalente à celle de Hamlet sur l'action, concerne en première place l'acte primordial de remplir la page:

Mon ame a refusé cent fois obeyssance à ce mien dessein d'escrire un mot sur les Essays...Lecteur, n'accuse pas de temerité le favorable jugement qu'il a faict de moy, quand tu considereras, en cet escrit icy, combien je suis loing de le meriter (51).

Étant donné une telle absence de validité dans le véhicule médiateur de la 
subjectivité, c'est-à-dire dans l'écriture, la comparaison et la ressemblance deviennent pour Marie de Gournay le champ véritable de la lutte existentielle, comme c'est le cas chez Hamlet. En plus, pour celui-ci, ce ne sont pas seulement les fréquents soliloques, opposés à l'action, qui marquent un discours fortement subjectif, mais aussi la lutte à la fois pour et contre l'intérêt paternel qui est souvent représentée en termes qui relèvent de l'écriture. ${ }^{15}$

Dans cette optique, il parait significatif que la veuve de Montaigne soit représentée par de Gournay d'une manière psychologiquement compliquée, à la différence d'une autre veuve, Madame d'Estissac, qui figure comme dédicataire de l'essai mentionné plus haut: "De l'Affection des pères aux enfans." Montaigne suit une formule assez conventionnelle pour louer Madame D'Estissac en tant qu'échantillon de veuvage noble et "d'affection maternelle" (2:59) — un statut qui dépend apparemment de son refus des "grands et honourables partis qui vous ont esté offerts" (58). Les valeurs témoignées par l'auteur correspondent assez étroitement àcelles du héros shakespearien, pour lequel le mariage de sa mère avec Claudius, le nouveau roi, la rend à ses yeux déloyale envers feu son père et, par extension, envers lui-même.

Mais c'est chez Marie de Gournay que l'on trouve une projection d'émotion presque aussi intense que dans le cas de Hamlet, sur la survivante - celle qui représente le seul lien avec un "père" idéalisé mais qui a également eu une relation intime et privilégiée avec le défunt. Ce dernier aspect suggère forcément la présence de la jalousie sous la forme de l'identification. (Chez Hamlet, une jalousie pareille est évidente dans son hostilité envers Claudius, laquelle $\mathbf{a}$ un caractère obsessionnel et franchement sexuel). En établissant le dévouement absolu de Madame de Montaigne, qui a "rendu les offices d'une tres-ardente amour conjugale à la memoire de son mary" (25) - précisément l'idéal que Hamlet, plein d'amertume, accuse sa mère d'avoir violé — de Gournay anticipe nettement la description, déjà citée, de ses propres sentiments comme à la fois "amy" et "fille." Elle commence par l'image des larmes - également la préoccupation de Hamlet, dont la mère s'était d'abord comportée "Like Niobe, all tears" (1.2.49) avant de révéler ses larmes comme étant "unrighteous" (154). Marie de Gournay pose la question:

Qualifierons nous ces larmes odieuses ou desirables? veu que, si Dieu l'a reservée au plus lamentable des veufvages, il luy a pour le moins assigné quand et quand en luy le plus honnorable tiltre qui soit entre les femmes? Et n'est Dame de merite et de valeur, qui n'aymast mieux avoir eu son mary, qu'en avoir nul autre, tel qu'il soit. Haut et glorieux advantage que le pis, dont Dieu l'ait estimée digne, reste encore achetable au pris de toute autre felicité (26). 
Voilà que Hamlet se met au carrefour intertextuel de de Gournay et de Montaigne, puisque celui-ci, d'après Ovide, cite Niobe comme pétrifiée à la suite de la perte de ses enfants, plutôt que comme veuve pleurante. ${ }^{16}$

Encore une fois, cette pulsion vers l'identification pousse de Gournay dans deux directions, selon un procédé qui correspond aussi à la situation dans la pièce, où le héros se regarde simultanément dans la position de sa mère et celle de son père. Car grâce aux sentiments purement dévoués de Madame Montaigne, au contraire notamment de Gertrude, de Gournay peut réussir à prendre dans un sens la place du mari, comme voudrait le faire Hamlet, selon le complexe d'Oedipe souvent invoqué dans son cas. Nous avons déjà remarqué que Marie de Gournay témoigne de sa gratitude à la veuve "d'avoir voulu r'embrasser et r'échauffer en moy les cendres de son mary, et non pas l'espouser mais se rendre une autre luy-mesme." Elle développe sa pensée en représentant cette dame comme "ressuscitant en elle à son trespas une affection où jamais elle n'avoit participé que par les oreilles, voire luy restituer un nouvel image de vie par la continuation de l'amitié qu'il me portoit" (26).

$\mathrm{Si}$, du point de vue intertextuel, on peut donc considérer le texte de Marie de Gournay comme une sorte d'activeur de certains éléments montaigniens, activeur qui rend psychologiquement plus puissants leurs équivalents dans l'oeuvre de Shakespeare, il est néanmoins remarquable de trouver au coeur de la Préface la question centrale à la fois pour Montaigne et Hamlet, celle de l'être, explicitement traitée d'une façon qui ajoute encore la dimension de la comparaison, du miroir. Car quand Marie de Gournay reprend le principe de Montaigne que "estre consiste en mouvement et action," elle applique cette idée à sa propre situation vis-à-vis de son "père," la position équivoque de "semblable" sur laquelle elle base son droit d'écrire, équivalent pour elle à agir, donc à être: "les grands esprits sont...affolez des grands esprits: comme tenans leur estre du mouvement, et leur prime mouvement de la rencontre d'un pareil" (47). Et un peu plus loin on lit aussi: 'Estre incognu c'est aucunement n'estre pas; car estre se refere à l'agir; et n'est point, ce semble, d'agir parfaict, vers qui n'est pas capable de le gouster" (48). Encore deux fois dans cette page de Gournay se sert du mot "semblable," en parlant d'abord de "la volupté de l'esprit qui naist principalement en ce commerce d'un semblable," puis du besoin de "quelque suffisance semblable" pour communiquer nos "belles conceptions" (48).

Une concordance ${ }^{17}$ nous permet de constater que Montaigne utilise luimême le mot "semblable" assez rarement dans les Essais: comme substantif, 
trois fois seulement et jamais au singulier. Or Shakespeare emploie ce terme d'origine française - attesté cependant en anglais dès le quinzième siècle, seulement deux fois: dans Timon of Athens, qui date de 1608 environ, et dans Hamlet, pour le discours de son héros. Ici Hamlet se moque d'un certain courtisan Osric, qui vient de faire l'éloge de Laertes en termes affectés, précieux: "to make true diction of him, his semblable is his mirror, and who else would trace him, his umbrage, nothing more" (5.2.118-20). En fait, le portrait de cet Osric à été bien tracé par Marie de Gournay, lorsqu'elle défend Montaigne contre ceux qui l'accusent d'avoir introduit de nouveaux mots:

...quelques jeunes courtisans...ne cherchent pas d'innover pour amender, mais d'empirer pour innover: et, qui pis est, avec condemnation des vieux vocables, qui sont ou meilleurs ou, s'ils sont egaux, doivent encore estre preferez par l'usage (30-31).

Toutefois certains de ces soi-disant innovateurs linguistiques, affirme-t-elle, "se moqu[ent] bien de nostre sottise à nous autres, quand nous dirons: son 'lever,' son 'col,' sa 'servitude,' au lieu de leurs nouveaux termes: son 'habiller,' son 'coulx,' son 'esclavitude,' et semblables importantes corrections" (31). De Gournay semble anticiper à la fois le caractère d'Osric et l'esprit moqueur de Hamlet.

Mais ce qui compte encore plus, c'est que l'idée du semblable devient effectivement de plus en plus pressante pour Hamlet, malgré l'ironie dont il charge le mot. À propos de Laertes, qui réussira bientôt comme vengeur de son propre père aux dépens de Hamlet, le Prince vient de dire:

But I am very sorry, good Horatio,
That to Laertes I forgot myself,
For by the image of my cause I see
The portraiture of his.

Plus tôt, il y avait eu un autre quasi-vengeur de père, Fortinbras, à qui Hamlet s'était comparé. (Celui-là est maintenant en passe de réussir, lui aussi, tout en prenant la place de Hamlet sur le trône). Mais c'est surtout du dénommé Horatio, son seul ami fiable et dévoué, que le héros cherche à faire un semblable de lui-même presque tout au long de la pièce, en idéalisant ses qualités personnelles. Et dans le dialogue que je viens de citer, Hamlet lui fait part de sa "conscience" pour justifier sa nouvelle résolution: "is't not perfect conscience/To quit him with this arm? (5.2.67-68). Cette résolution d'un débat intérieur manifeste une des marques d'un "homme parfaict," d'après Marie de 
Gournay, c'est-à-dire de "sçavoir où la vengeance est licite" (37), et illustre à la perfection l'affirmation de celle-ci qu'"un sage languit si'l ne peult rendre un homme de bien tesmoign de la pureté de sa conscience" (48).

Finalement, Hamlet s'appuie sur Horatio pour raconter son histoire après sa mort: "O God, Horatio, what a wounded name,/Things standing thus unknown, shall I leave behind me" (5.2.345). Voire, à ce moment, pour lui "Estre incognu c'est aucunement n'estre pas." Il se sent obligé de condamner Horatio, qui ne veut pas survivre à son ami, à "La plus grande infelicité du monde," en lui demandant:

If thou didst ever hold me in thy heart,

Absent thee from felicity a while,

And in this harsh world draw thy breath in pain

To tell my story.

Marie de Gournay avoue qu'une telle pulsion de se faire connaître auprès de ce "qui [est] capable de le gouster" peut se qualifier d" "ambition" (48). L'intertexte jette ainsi une lumière nouvelle sur la discussion floue de son ambition où Hamlet permet aux faux amis Rosencrantz et Guildenstern de l'engager dans le deuxième acte (2.2.252 ff.) et encore plus tard (3.2.340 ff.).

La mention de ce "wounded name" renvoie au soliloque déjà cité dans lequel Hamlet déplore le fait que trop réfléchir - ce qu'il appelle "conscience" - interrompt ce mouvement en qui consiste l'être, de sorte que nos grands projets "lose the name of action." La plupart des spécialistes shakespeariens semblent avoir négligé le fait que "name" dans ce contexte veut dire "renommée," comme c'est souvent le cas dans l'anglais de l'époque. Au bout du compte, la contribution clé de l'intertexte gournayen à la lecture de Hamlet est peut-être d'introduire ce terme de "renommée" comme médiateur, pour ainsi dire, dans l'équation entre "estre" et "action" proposée par Montaigne. Bien entendu, il s'agit de la renommée dans un sens large et complexe. Paradoxalement, c'est en tant que championne de Montaigne que Marie de Gournay doit s'opposer dès le début de sa Préface à l'opinion de Montaigne même que "la renommée de ce livre suffit à son merite" (de Gournay 23), et la démarche de ses pensées établit que, pour elle, l'idée de la renommée s'éloigne de cette qualité inerte et indépendante qui figure dans de nombreuses allégories du Moyen Age et de la Renaissance. Du point de vue de Marie de Gournay, la renommée se fait à deux dans un processus qui dépend de "l'alliance d'une autre" (47-48), le "commerce d'un semblable" (48). Comme le révèle le mot "alliance," il s'agit avant tout de définir son propre rôle de 
"fille" dans le drame qui se déroule autour de la mémoire de son "père." En lui conférant plus de renommée, elle se renomme littéralement, de telle sorte qu'elle s'exempte d'être nommée par les hommes comme dans l'équation misogyne de Hamlet: "Frailty, thy name is woman!"

Ainsi l'élément de renommée entraîne pour Marie de Gournay, comme pour Hamlet, tout un système de relations personnelles, fondé sur la notion, si instable et fuyante qu'elle soit, du "semblable." Dans la pièce de Shakespeare, cette idée fait sa première apparition dès le moment où Hamlet, dans son premier soliloque, injure son nouveau "père" aussi bien, ironiquement, que luimême au moyen d'encore une autre équation: "No more like my father/Than I to Hercules" (1.2.152-53). Hercule - l'archétype de l'homme fort, de l'homme d'action, mais tout de même conquis par une femme, comme le sait bien Marie de Gournay lorsqu'elle se moque des vantards anti-féministes, en exposant leur propre "foiblesse" (27): "Cet autre, en fin, bravant une femme, fera cuider à sa grand' mere que, s'il n'estoit pitoyable, Hercules ne vivroit pas" (28). Le triomphe d'Omphale, lequel sera cité par Marie de Gournay dans son ouvrage de 1622, Egalité des hommes et des femmes, fait également partie de la légende. ${ }^{18}$ Ainsi par la voie intertextuelle le facteur féminin, celui de "gaingiving," gâte encore des équations masculines tranchées, binaires, en parlant, même si c'est à voix basse, des courants confus et contradictoires de l'esprit. L'idée d'un semblable parfait qui garantira la renommée, donc l'être, doit rester inachevée, étant donné qu'en même temps, comme le "père" l'a bien appris à sa "fille d'alliance," "la vie mesme n'est qu'une contexture de punctilles" (de Gournay 42). Il ne nous en reste pas moins l'impression que prêter l'oreille à cette voix féminine aurait pu sauver la vie de Hamlet, même au prix de la lui avoir rendue encore plus impossible.

University of Western Ontario

\section{Notes}

Note: Je tiens à remercier le Professeur François Rigolot d'avoir relu le manuscrit de cet article et fait des suggestions précieuses.

1. Voir mon ouvrage, Intertextuality and Romance in Renaissance Drama: The Staging of Nostalgia (Basingstoke, Hampshire, Macmillan, et New York, St. Martin's, 1992).

2. William Shakespeare, The Riverside Shakespeare, éd. G. Blakemore Evans et al. (Boston, Houghton Mifflin, 1974).

3. Pour un survol très bien effectué de ce domaine, voir Robert Ellrodt, "Self-Consciousncss in Montaigne and Shakespeare," Shakespeare Survey, 28 (1975), 37-50. 
4. Voir Ellrodt, 40.

5. Montaigne, Essais, sous la direction de Maurice Rat, 3 vols. (Paris, Garnier, 1957).

6. John W. Robertson, Montaigne and Shakespeare, and Other Essays on Cognate Questions, $2^{c}$ éd. (Londres, Black, 1909), pp. 61-62; sur l'utilisation du mot "discours" chez Montaigne et Shakespeare, voir aussi pp. 46-48.

7. Pourtant il s'agit pour Robertson de l'influence supposée de la traduction anglaise de Florio. Dans ce cas, comme dans bien d'autres, la traduction n'est pas plus proche du texte shakespearien que l'original.

8. Voir Cathleen M. Bauschatz, "Imitation, Writing, and Self-Study in Marie de Gournay's 1595 'Préface' to Montaigne's Essais," dans Marie-Rose Logan and Peter L. Rudnytsky, eds. Contending Kingdoms: Historical, Psychological, and Feminist Approaches to the Literature of Sixteenth-Century England and France (Detroit, Wayne State University Press, 1991), pp. 348-49.

9. Bauschatz, p. 349.

10. François Rigolot, Introduction, Marie de Gournay, "Préface à l'édition des Essais de Montaigne," sous la direction de François Rigolot, Montaigne Studies: An Interdisciplinary Forum, 1 (novembre 1989), 17.

11. Rigolot, Introduction, 17.

12. Marie de Gournay, "Préface à l'édition des Essais de Montaigne," sous la direction de François Rigolot, Montaigne Studies: An Interdisciplinary Forum, 1 (novembre 1989), 7-20.

13. Rigolot, Introduction, 16n18, renvoyant à son article, "L'amitié intertextuelle: Etienne de La Boétie et Marie de Gournay," dans L'Esprit et la Lettre: Mélanges offerts à Jules Brody (Tübingen, Narr, 1991), pp. 57-66, dans lequel il souligne l'intérêt de Gournay pour la "crédibilité de [s]a succession" (58) à Montaigne et relit de façon convaincante "ce nouveau discours de l'amitié de Marie de Gournay à la lumière du célèbre chapitre 28 du premier livre des Essais" (59).

14. Voir Terence Cave, The Cornucopian Text: Problems of Writing in the French Renaisance (Oxford, Clarendon Press, 1979), p. 274, et Philippe Desan, "The Book, the Friend, the Woman: Montaigne's Circular Exchanges," trad. Brad Bassler, dans Marie-Rose logan and Peter L. Rudnystky, eds. Contending Kingdoms: Historical, Psychological, and Feminist Approaches to the Literature of Sixteenth-Century England and France (Detroit, Wayne State University Press, 1991), pp. 225-62.

15. Voir Jonathan Goldberg, "Hamlet's Hand," Shakespeare Quarterly, 39 (1988), 307-27.

16. Ce passage m'a été signalé par François Rigolot, qui propose, dans Les métamorphoses de Montaigne (Paris, Presses Universitaires de France, 1988), que cette allusion constitue "la figure emblématique de l'écrivain lui-même que, ayant perdu en La Boétie son idéal soutien, reste pétrifié à jamais dans sa douleur infinie" (223).

17. Roy E. Leake, Concordance des Essais de Montaigne, 2 vols. (Genève, Droz, 1981).

18. Voir Rigolot, Introduction, $28 \mathrm{n} 19$. 